\title{
APLICACIÓN DE SISTEMAS DE CALIDAD EN LA ELABORACIÓN DE ALIMENTOS Y SERVICIOS AL CLIENTE PARA ESTABLECIMIENTOS DE EXPENDIO DE COMIDA NO CLASIFICADOS EN EL DISTRITO DE JESÚS MARÍA
}

\author{
Walter David Ugarte Casafranca* \\ Luis Álvaro Bueno Acosta**
}

RESUMEN

En el Perú existe un divorcio entre su riqueza gastronómica y los parámetros o estándares mínimos de calidad que garanticen un óptimo servicio en los restaurantes. La finalidad de la aplicación de los Planes de Calidad HACCP es crear sellos de garantía que emitan una nueva imagen de calidad gastronómica peruana y desarrollar herramientas de gestión empresarial que mejoren el producto para el consumidor.

Con la presente investigación se intenta proponer mecanismos que permitan mejorar el servicio de los restaurantes, basando dichos mecanismos en los sistemas internacionales HACCP, adaptándolos a nuestra realidad y proponiendo que se creen sellos de garantía para los establecimientos de expendio de comida no clasificados en el distrito de Jesús María.

Palabras clave: alimentos, calidad, restaurantes, HACCP

\begin{abstract}
In Peru there is a divorce between its rich cuisine and parameters or minimum quality standards to ensure optimum service in restaurants. The purpose of the application of HACCP Quality Plans is to create security seals in order to spread a new image quality Peruvian cuisine, and develop business management tools that improve consumer product.

With this research we attempt to propose mechanisms to improve the service of restaurants, basing these mechanisms on HACCP international system, adapted to our reality and proposing the creation of guarantee seals for this establishments in Jesus Maria district.
\end{abstract}

Keywords: food, quality, restaurants, HACCP

\footnotetext{
* Abogado, Docente de la Facultad de Ciencias Administrativas de la UNMSM, Gerente General del Centro de Producción Editorial e Imprenta de la UNMSM

** Bachiller en Administración de Turismo y Hotelería, egresado de la Facultad de Ciencias Administrativas de la Universidad Nacional Mayor de San Marcos
} 


\section{INTRODUCCIÓN}

El sistema HACCP (Análisis de Peligros y Puntos Críticos de Control) es un sistema desarrollado hace más de tres décadas con el fin de brindar alimentos seguros a los astronautas del programa espacial de la NASA, y en la actualidad es un programa ampliamente empleado por las industrias de alimentos. El objetivo principal es garantizar la seguridad del producto.

Dentro del distrito de Jesús María, Lima, de un registro de 1352 mypes, se establece que 717 son de actividades comerciales, 602 son de actividades de servicios y solo 33 son de actividades productivas. Dentro de las actividades servicios se tiene un aproximado de 300 establecimientos de expendio de alimentos, los cuales por su naturaleza de ser empresas mypes sufren de una serie de limitaciones para su desarrollo, estas se encuentran en el orden de la informalidad, desarticulación empresarial, dificultad de acceso al financiamiento, falta de información y limitadas capacidades operativas y gerenciales entre otras. Como si esto fuera poco, los restaurantes en particular deben desplegar estrategias para atraer a sus potenciales clientes con un servicio de calidad, que comprenda condiciones de higiene, inocuidad de los alimentos y una atención orientada a cubrir las expectativas de los mismos. La integración de estas cualidades permitiría a los restaurantes del distrito contar con una herramienta nueva y muy competitiva para mejorar su imagen y por ende su desarrollo empresarial.

Cuando se desea identificar la calidad de servicio de un restaurante se ha reconocido de manera institucional la clasificación por número de tenedores que otorga vía sus dependencias regionales el Mincetur. En la práctica, esta calificación lamentablemente solo alcanza a un número mínimo de restaurantes, preferentemente anexados a una firma hotelera internacional o bien que se ubican en lugares muy turísticos, además dicha clasificación es muy genérica dentro de los aspectos de seguridad alimentaria y define la categoría que puede ostentar un establecimiento en función de variables, tales como la infraestructura, equipos, capacidad instalada, cartas, personal, entre otras. Definitivamente dicho cuadro de clasificación no responde a la realidad de la gran mayoría de restaurantes en el Perú, ni mucho menos sirve como una herramienta de medición de los productos y servicios que se brindan en los establecimientos de expendio de comidas.

\section{METODOLOGÍA}

\section{Formulación del problema}

¿Puede tomarse en consideración los modelos del sistema HACCP en los establecimientos de expendio de comida del distrito de Jesús María para garantizar la mejora de la calidad de dichos establecimientos?

\section{OBJETIVOS DE LA INVESTIGACIÓN}

\section{Objetivo general}

Proponer mecanismos que mejoren la calidad de servicios que se brindan en el tema de restauración, basado en los sistemas de calidad existentes como el HACCP, para los establecimientos de expendio de comida no clasificado en el distrito de Jesús María.

\section{ESTUDIO DEL DISTRITO DE JESÚS MARÍA}

A comienzos del siglo XX, el desarrollo urbano en Lima comenzó a extenderse y abrió paso a la fundación de un próspero barrio residencial de hermosas casonas, cuya belleza arquitectónica, ha marcado un estilo colonial que aún persiste. Llegó la luz eléctrica, las redes de agua potable y la vida de los vecinos de lo que actualmente es el distrito de Jesús María cambió radicalmente.

Equidistante a todos los lugares de la provincia de Lima, Jesús María es uno de los 43 distritos de la capital del Perú mejor ubicados. Cuenta con un clima templado, de temperaturas que oscilan entre los 14 grados en invierno y 28 grados en verano.

En estas áreas, también se construyeron importantes edificaciones como el Club Lawn Tennis, el Jockey Club, el Hipódromo de Santa Beatriz y las amplias residencias de la época eran consideradas las mejores en todo Lima. Posteriormente, en lo que fue el "Bosque Matamula" se construyó el Hospital "Edgardo Rebagliati Martins", perteneciente al entonces Seguro Social del Empleado, el cual pasó a llamarse Instituto Peruano de Seguridad Social y actualmente se denomina EsSalud.

\section{EVALUACIÓN SOCIOECONÓMICA}

El distrito de Jesús María ha pasado por un proceso de ordenamiento y formalización de las actividades empresariales comerciales, lo que ha permitido una importante participación de la Inversión Privada en comercio y vivienda. 
De acuerdo a los informes de SUNAT, Jesús María se encuentra en:

- El segundo lugar en actividades relacionadas al campo médico, odontológico, así como en instituciones superiores y universidades

- $\quad$ El tercer lugar, luego del Cercado y San Isidro en actividades de contabilidad, asesoramiento empresarial, actividades jurídicas, panadería, imprenta, investigación, vigilancia, construcción de viviendas, agencias de empleos, agencias de viajes e inmobiliarias.

La Comisión de Promoción de la Pequeña y Microempresa - PROMPYME, en el año 2003 realizó un estudio en un grupo de restaurantes de Lima Metropolitana, a partir del cual identificó las deficiencias y problemas básicos relacionados con la manipulación de alimentos en estos establecimientos.

- Se cumple solo en un $43 \%$ las buenas prácticas de elaboración de alimentos.

- Se cumple solo en un 56\% las prácticas de saneamiento básico.

- Se cumple solo en un 57\% los requisitos mínimos de infraestructura del establecimiento.

- $\quad$ Se cumple en un 70\% las medidas de seguridad.

\section{BASES LEGALES}

\section{Normativa internacional}

- Codex Alimentarius

- $\quad$ Food \& Drug Administration - FDA

- International Commission on Microbiological Specifications for Foods

- $\mathrm{HACCP}$

- Organización Mundial de la Salud - OMS

- Organización Panamericana de la Salud - OPS

\section{NORMATIVA NACIONAL}

\section{Gobierno Central:}

- $\quad$ Ley 28015 - Ley de Pequeña y Micro Empresa

- $\quad$ Ley 26842 - Ley General de Salud

- Decreto Supremo N. ${ }^{\circ}$ 007/98/SA - Reglamento sobre vigilancia y control sanitario de alimentos y bebidas y de creación del Comité Nacional del Codex

- Norma Sanitaria para el funcionamiento de los restaurantes y servicios afines $N .^{\circ} 363-2005$ / MINSA

- Criterios Microbiológicos de Calidad Sanitaria e Inocuidad para los Alimentos y Bebidas para Consumo Humano R.M N. ${ }^{\circ}$ 615- 2003 / SA/DM

- Reglamento Sanitario para el Funcionamiento de Autoservicios de Alimentos y Bebidas RM N. ${ }^{\circ} 1653-2002-/$ SA /DM

- $\quad$ Decreto Supremo N. ${ }^{\circ}$ 025-2004-MINCETUR

Gobierno Local

- Ley 27972 - La nueva Ley de Municipalidades

- Ordenanza N. 550 - Sistema Metropolitano de Supervisión y control de alimentos y bebidas de consumo humano (SIMSCABH)

- $\quad$ Ordenanza N. ${ }^{\circ}$ 082-MLM - Ordenanza de Salud y Salubridad Municipal

- Ordenanza N. ${ }^{\circ} 141$. Ordenanza sobre obligatoriedad de portar Carné de Salud

\section{HIPÓTESIS}

Si se tomara en consideración los modelos del sistema HACCP en los procesos de producción y atención de los servicios de alimentación en los establecimientos de expendio de comida no clasificados de Jesús Maria, entonces se mejoraría la calidad de los alimentos, la percepción y satisfacción de los consumidores, comprometiendo a los municipios en su aplicación.

\section{DISEÑO METODOLÓGICO}

La presente investigación es de carácter descriptivo, exploratorio; participando 90 empresas de restaurantes del distrito de Jesús María.

\section{POBLACIÓN OBJETIVO}

Se consideró en la muestra a 90 establecimientos de expendio de comidas no clasificados del distrito de Jesús María.

\section{UNIDAD DE MUESTREO}

\section{Criterios de inclusión}

- Restaurantes no clasificados ubicados en el distrito de Jesús María.

- Restaurantes de comida peruana (criolla, pescados y mariscos y menú).

- Pollerías y anticucherías (pollos y parrillas). 
- Restaurantes de comida china (chifa).

- Restaurantes de comida internacional (comida italiana, entre otros).

- Fuentes de soda y cafeterías (juguerías y bares).

- Otros (comida naturista, otros).

\section{TIPO DE ESTUDIO}

El tipo de estudio de investigación es de carácter exploratorio, de acuerdo al tipo de empresa, estructura de la organización y condiciones de manipulación de alimentos.

\section{PROPUESTA DE APLICACIÓN DEL SISTEMA HACCP}

\section{Significado de HACCP}

El HACCP (del inglés Hazard Analisis and Critical Control Point) es un enfoque sistemático que con base científica nos permite identificar riesgos específicos y medidas de control con el fin de asegurar la inocuidad de los alimentos. El HACCP es un instrumento para evaluar riesgos y establecer sistemas de control que se orientan hacia la prevención en lugar de basarse en el análisis del producto final. También se puede definir como una estrategia de aseguramiento de la calidad preventiva dirigida a todas las áreas de contaminación, supervivencia y crecimiento de microorganismos.

El HACCP fue desarrollado en el año 1960 por la Pillisbury Company, a solicitud y con el apoyo de la NASA (National Aeronautic and Space Administration) y Natick Laboratory (de la Armada de los Estados Unidos) y el Air Force Space Laboratory Project Group. El objetivo primordial era producir un alimento con el $100 \%$ de seguridad y que pudiera ser usado por astronautas del naciente programa espacial, sin peligro de ser infectados por contaminantes químicos, toxinas o microorganismos patógenos.

\section{Principios en que se basa el HACCP}

Estos principios son aceptados ampliamente y deben seguirse para elaborar un plan de HACCP, ya que explican con detalle el proceso.

Principio 1: Enumerar todos los peligros posibles relacionados con cada etapas realizando un análisis de los peligros, a fin de determinar las medidas para controlar los peligros identificados.

Principio 2: Determinar los Puntos Críticos de Control (PCC).
Principio 3: Establecer el límite o los límites críticos (LC) en cada Punto Crítico de Control (PCC).

Principio 4: Establecer un sistema de vigilancia del control de los Puntos Críticos de control (PCC).

Principio 5: Establecer medidas correctivas que han de adoptarse cuando la vigilancia indica que un determinado Punto Crítico de Control (PCC) no está controlado.

Principio 6: Establecer procedimientos de verificación o de comprobación para confirmar que el sistema HACCP funcione eficientemente.

Principio 7: Establecer un sistema de registro y documentación sobre todos los procedimientos y los registros apropiado para estos principios y su aplicación.

\section{ANÁLISIS Y PRESENTACIÓN DE RESULTADOS}

\section{Discusión y resultados}

La presente investigación permitirá concretar la implementación de dos líneas de acción: Plan de Higiene y BPM, que son esenciales en la aplicación de un sistema HACCP; el escenario serán los restaurantes no clasificados del distrito de Jesús María. Resaltando la organización que refuerce el futuro escenario en la implementación de normas y estándares de calidad.

En el plan de desarrollo concertado de Jesús Maria 2005 - 2015, se tiene registrado un universo aproximado de 300 restaurantes, de los cuales se extrajo una muestra representativa del $30 \%$.

Dado el número de empresas se sustenta que fundamentalmente son de estructura familiar, como se muestra en el Gráfico N. ${ }^{\circ} 1$ y el otro porcentaje son de tipo asociativa en la que son dueños dos o más personas.

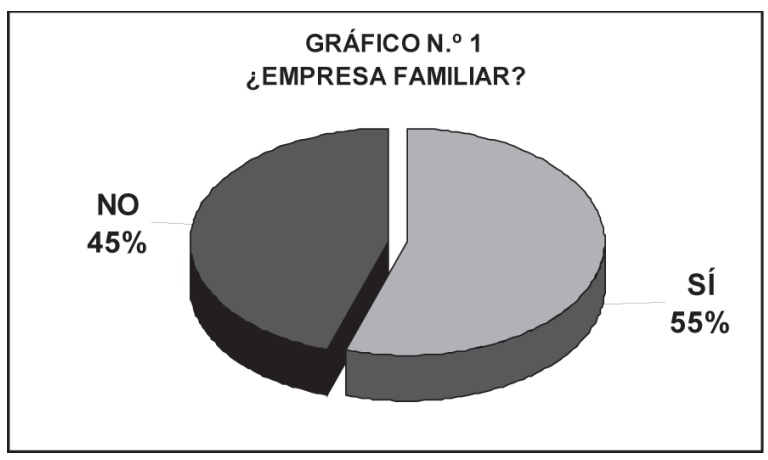

FUENTE: Elaboración propia. 
Organizativamente se sustenta en que la mayoría de las empresas se encuentran registradas en la modalidad de Persona Natural con un $80 \%$ y como persona Jurídica tan solo en un $20 \%$. Lo que nos indica que la formalización como empresa no se ajusta a las normas establecidas.

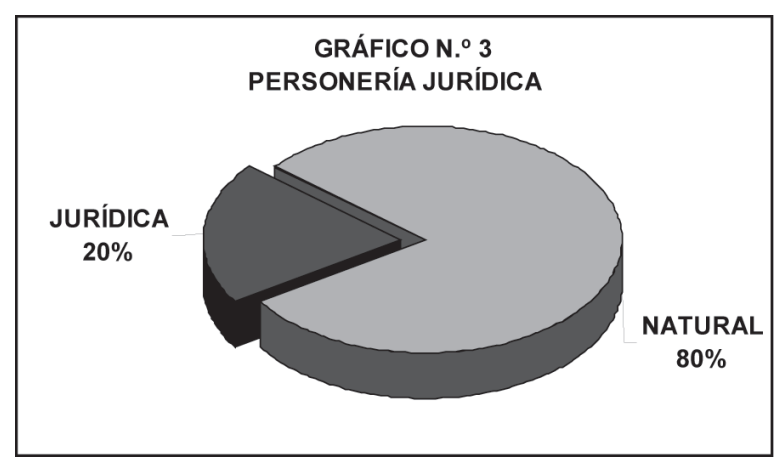

FUENTE: Elaboración propia.

En el plano de la formalización existe un alto grado de responsabilidad de cumplir con las normas establecidas con el municipio.

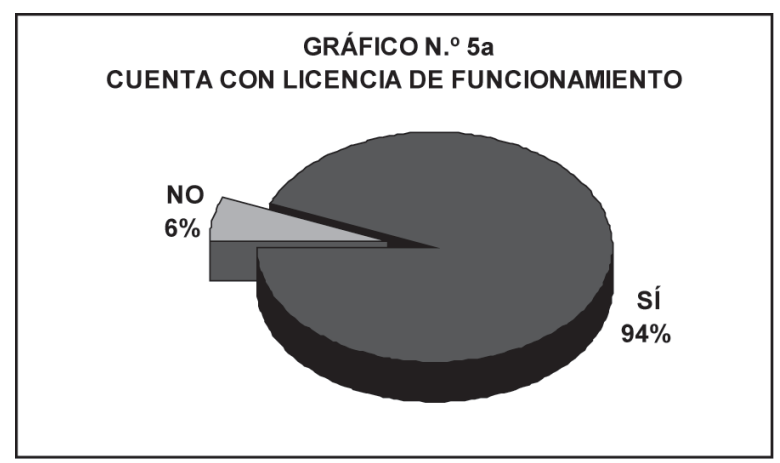

FUENTE: Elaboración propia.

La consolidación de los restaurantes no clasificados se puede visualizar en el $61 \%$ de licencias definitivas, lo que permite inferir que los negocios son rentables.

El 39\% de licencias provisionales responden a un mercado cambiante donde la apertura o cierre responde al mercado.

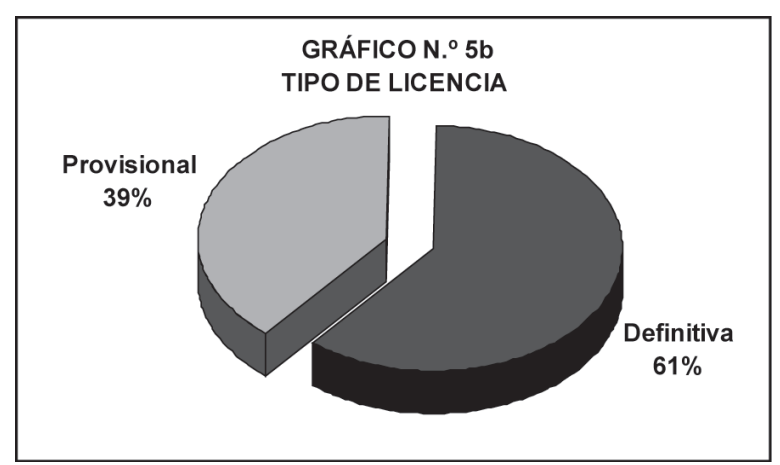

FUENTE: Elaboración propia.
La concentración de trabajadores en los establecimientos seleccionados permite Identificar que las empresas en nuestro universo son microempresas, fundamentada en la ley 28015 - Ley de Pequeña y Micro Empresa.

Por otra parte, encontramos que el número de trabajadores promedio que tienen la mayoría de empresas no alcanzan el número mínimo elemental de trabajadores que respondan a las actividades de manipulación, preparación, atención, limpieza y administración del local.

Esto se constituye en uno de los principales problemas para aplicar cualquier sistema de calidad.

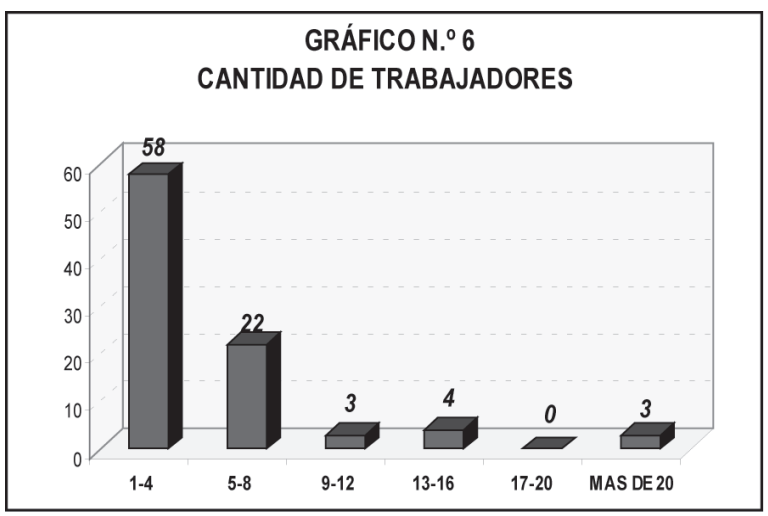

FUENTE: Elaboración propia

La importancia de ser parte de un Programa de implementación de HACCP se inicia con el compromiso del personal de la empresa, y uno de los primeros indicadores es contar con el carné de sanidad.

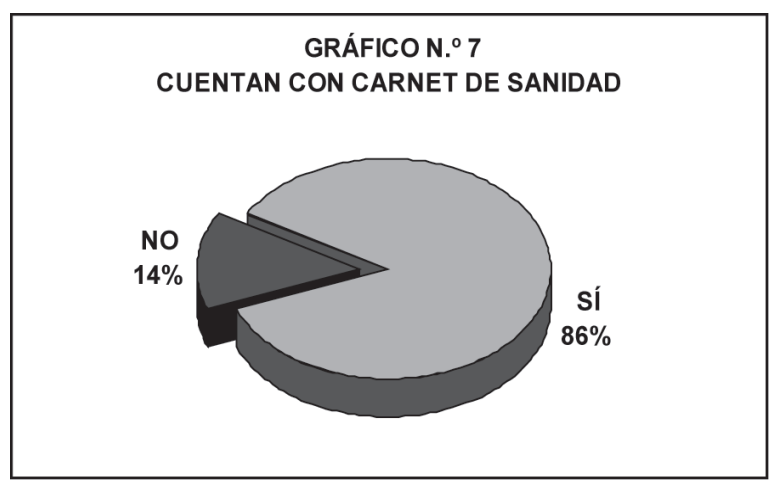

FUENTE: Elaboración propia

Es importante identificar la capacidad de los restaurantes para la atención a los clientes, en relación al volumen que se puede generar en un mismo momento, ya que eso nos permitirá proyectar los límites y a su vez el flujo de procesos para optimizar las diferentes actividades requeridas para el manejo de la empresa. 
Con lo antes dicho se puede concluir que las empresas de restaurantes en Jesús María están distribuidas en locales que tienen una capacidad máxima de 20 comensales hasta locales con una capacidad promedio que sobrepasa los 180 comensales.

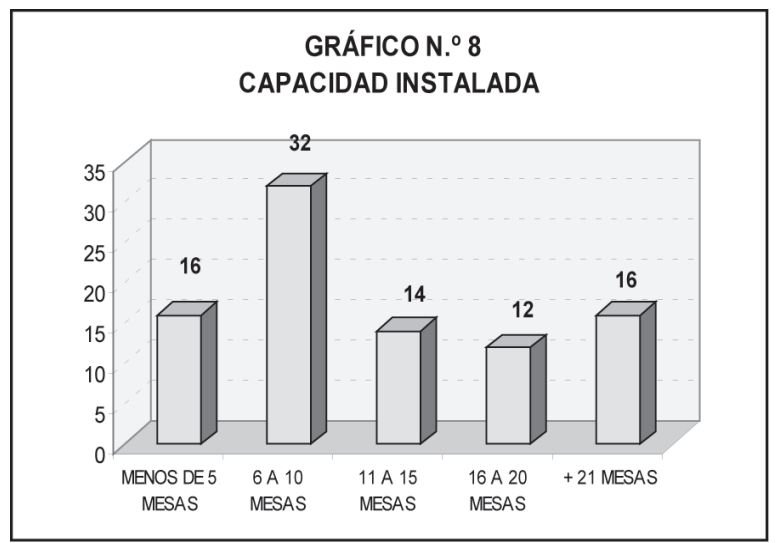

FUENTE: Elaboración propia.

La característica de todas las mypes es la presencia de empresas familiares y del compromiso del dueño en su negocio, la grafica muestra ese indicador como factor importante.

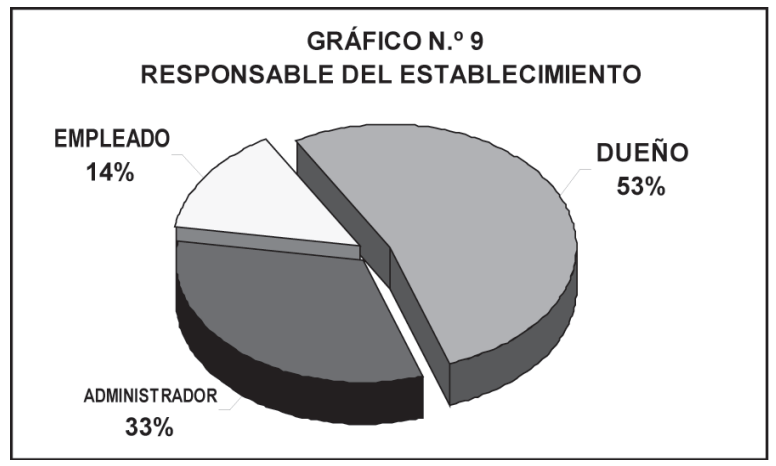

FUENTE: Elaboración propia.

Ser parte de un equipo HACCP implica seleccionar el personal adecuado que cumpla con la aplicación y desarrollo del sistema. Por ello, el grado de instrucción es un requisito indispensable.

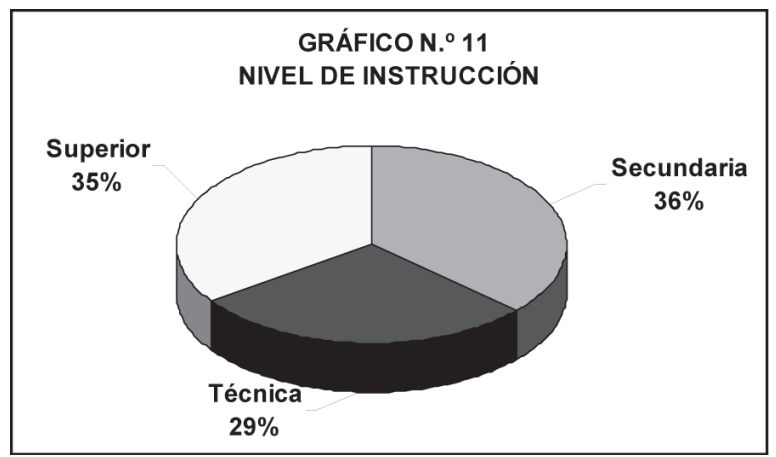

FUENTE: Elaboración propia.
La organización y puesta en marcha de un Sistema HACCP en un establecimiento de expendio de comida requiere que el responsable sea una persona preparada y de preferencia con experiencia en el área.

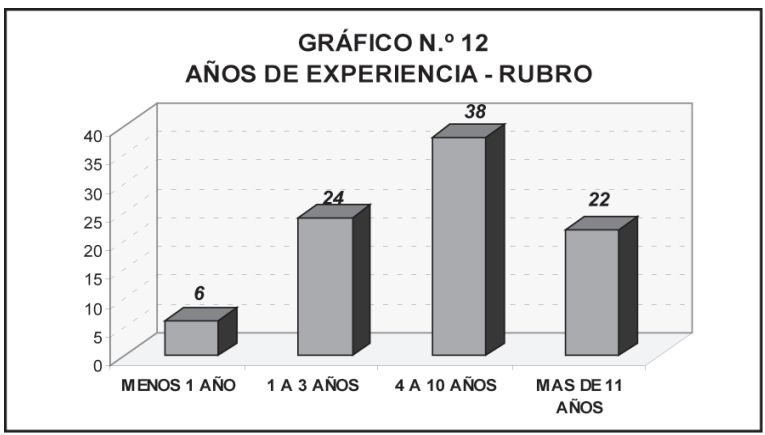

FUENTE: Elaboración propia.

La implementación de todo Plan HACCP requiere del interés y compromiso de los integrantes de la organización. Por lo anteriormente expuesto, es fundamental los programas de capacitación interna que programe la empresa.

En la gráfica, una primera incoherencia que implica desconocimiento ante lo encuestado y respondido es el cruce de indicadores: MANIPULACIÓN DE ALIMENTOS VS. BPM-BPH.

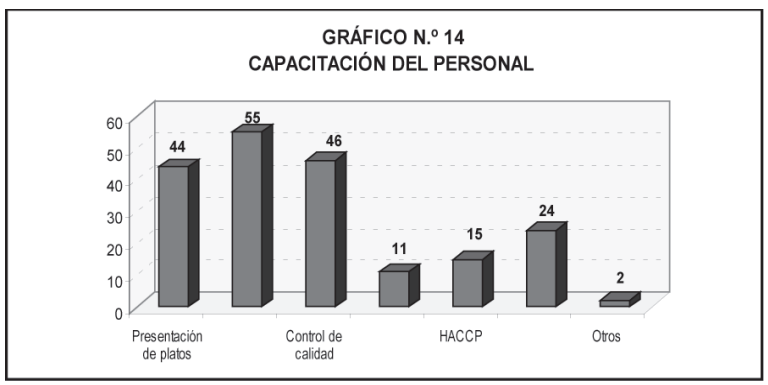

FUENTE: Elaboración propia.

El desarrollo de toda organización mype está fundamentada en el uso de los sistemas de información, en la gráfica se aprecia la carencia de la incorporación de sistemas informáticos, lo que a su vez no le permite técnicas ciertos procesos.

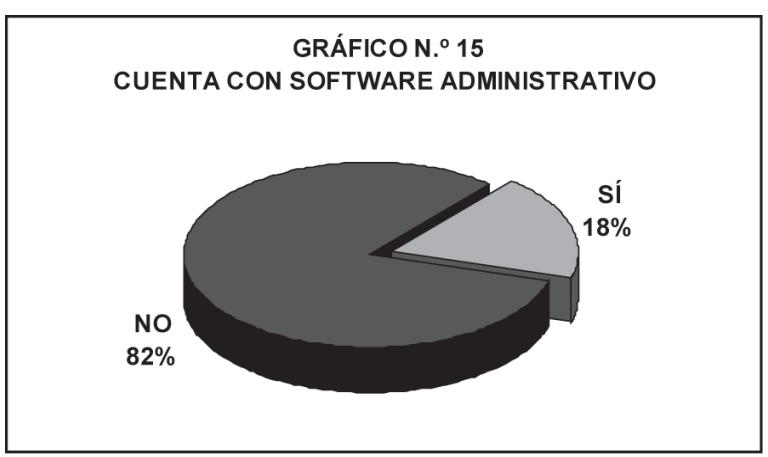

FUENTE: Elaboración propia. 
La organización debe seleccionar y asignar personal adecuado para asegurarse que las actividades que impactan sobre la no conformidad de los productos y servicios sean efectuados competentemente en base a la experiencia, capacitación, educación y habilidad, deberá incorporarse personal calificado y preparado para el logro de los objetivos del Plan HACCP.

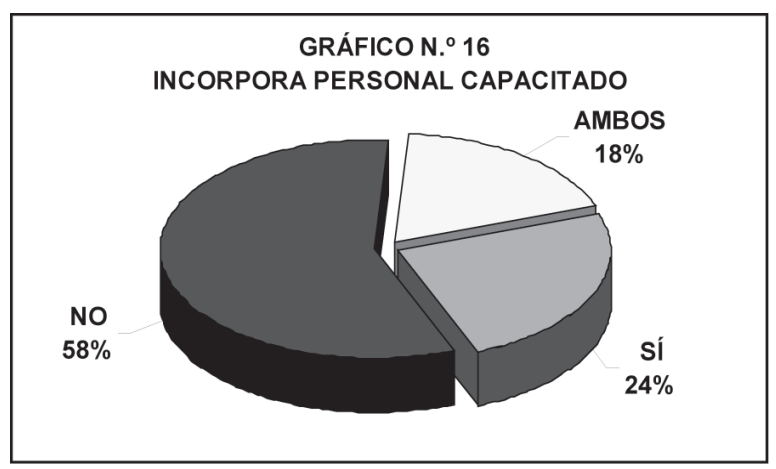

FUENTE: Elaboración propia.

En la presente gráfica observamos que en los restaurantes el personal trabaja con uniforme, lo que nos da una percepción de un intento de atención ordenada.

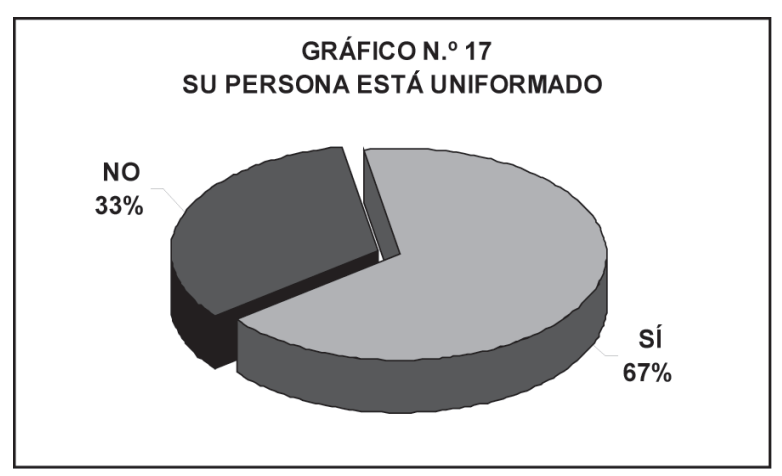

FUENTE: Elaboración propia.

La organización debe determinar y proveer los recursos necesarios para la implementación del Plan HACCP, y el presente gráfico muestra el interés de participar en un Programa de entrenamiento y nivel de competencia de los participantes.

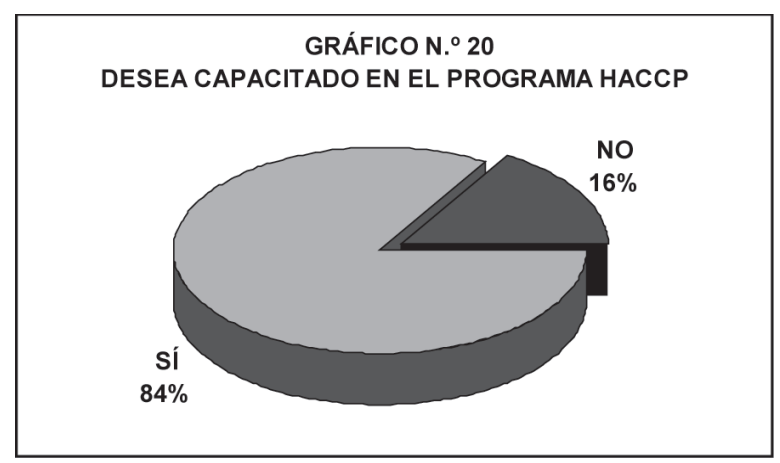

FUENTE: Elaboración propia.
De la muestra se aprecia que dentro de un marco de 1 a 5 años se encuentran la gran mayoría de maquinarias y equipos de los restaurantes encuestados.

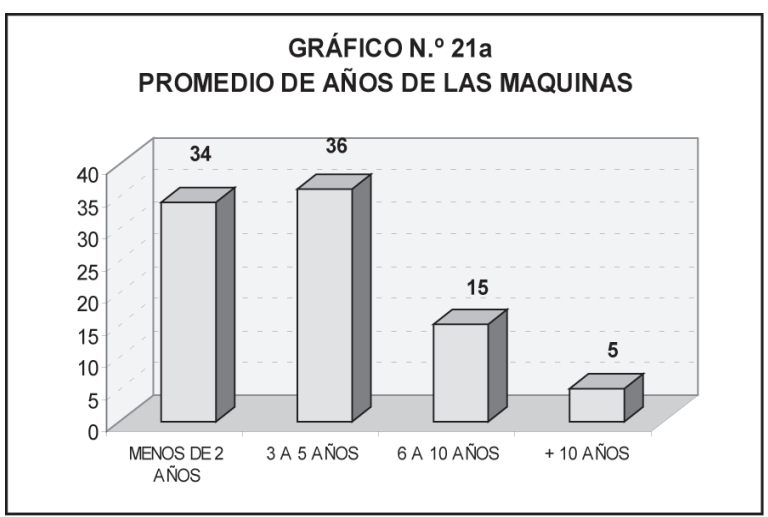

FUENTE: Elaboración propia.

En la muestra observada se aprecia un nivel de renovación de equipos alto si se toma en consideración que para estar en el campo de los que contestaron de manera afirmativa, tenían que haber renovado algún equipo en el presente año.

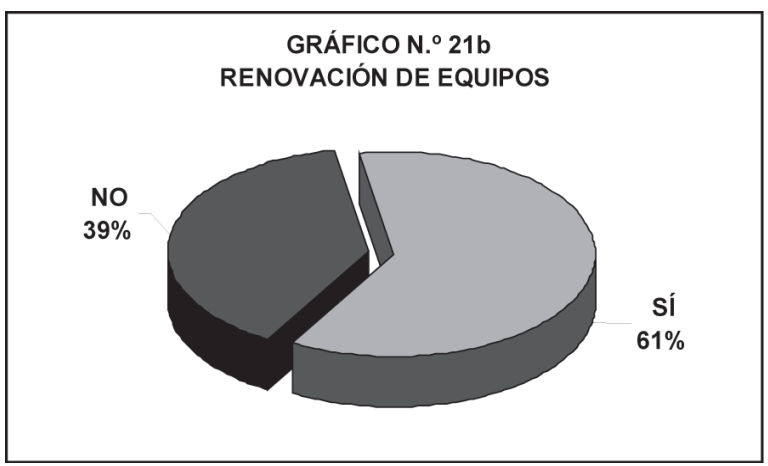

FUENTE: Elaboración propia.

\section{CONCLUSIONES}

1. La seguridad alimenticia no debe ser considerada como un valor agregado en los servicios de expendio de comidas, sino que es deber de quien los oferte como una característica implícita en el alimento que se produce.

2. La mayoría de empresas dedicadas al expendio de comidas, en el distrito de Jesús María no están constituidas como tal; debido al poco tiempo de posicionamiento en el mercado y para mayor comodidad desde el punto de vista legal y tributario responden como personas naturales.

3. La disposición a ser capacitados en sistemas de control de la calidad tales como el HACCP, identifican una tendencia a manejar estánda- 
res que les permitan un reconocimiento y una marca diferenciada, en el rubro de servicios.

4. La muestra seleccionada son mypes de servicios de expendio de comida que desean incorporar como una de las tareas de capacitación a su personal en las tecnología HACCP $(86 \%)$ - FACTOR COMPROMISO

5. Siendo su conformación de estructura organizativa familiar, generando empleo a sus grupos familiares empleos directos e indirectos - FACTOR DE GENERACIÓN DE EMPLEO.

6. La aplicación del sistema HACCP requiere de poca inversión en maquinaria y equipo, lo cual facilita la flexibilidad y adecuación con las mejoras e incorporación de los planes y programas HACCP, de acuerdo a las características propias del negocio.

7. El grado de instrucción superior de los responsables de los establecimientos de expendio de comidas acompañado a su juventud y años de experiencia se constituye en un factor positivo para la correcta aplicación del sistema HACCP.

8. La categorización de un establecimiento de expendio de comidas en el distrito de Jesús María no está regulada de manera correcta; lo que condiciona el desarrollo empresarial, pues no existe un sello de calidad que los identifique.

\section{RECOMENDACIONES}

Las tendencias actuales en el enfoque para conseguir la inocuidad de los alimentos muestran un escenario propicio para un uso extendido del Sistema HACCP en el futuro, como instrumento costo efectivo que permite la aplicación en diferentes eslabones de la cadena alimentaria, siendo una filosofía que hace de él una herramienta que contribuye a mejorar la eficiencia del proceso productivo de los alimentos y de la calidad del servicio.

La aplicación del sistema HACCP implica tomar en cuenta:

- Inversión vs. Mayores Ingresos

- Capacitación vs. Mejor servicio

- Tecnificación vs. Optimización de Procesos

- Orientación a la calidad vs.

\section{BIBLIOGRAFÍA}

Codex alimentarius commission committee on food hygiene. Guidelines for the application of hazard analysis critical control point (HACCP). system roma 2003 pp. 346 -67.

Comisión de análisis y evaluación del sistema de análisis y capacitación del sistema de análisis de riesgo y puntos críticos de control (HACCP) -manual de procedimientos: aplicación de sistema haccp senasa- GIPA 2001.

ROMERO, J. Puntos Críticos. Corporación Colombiana Internacional Santa Fe de Bogotá.

ALBRECH, KARL. La Excelencia del Servicio, ED. LEGIS BARCELONA 2000, 235 PP.

Binsa Patronato de Estudios Económicos y Sociales de La Industria de Restaurantes, Estudio del Mercado Nacional de Restaurantes.

BIOSCA, DOMENENC. 100 detalles que hay que cuidar en un restaurante. ED. LUISA, MÉXICO 1992, 164 PP.

Manejo Higiénico de Los Alimentos. ED. LIMUSA. MÉXICO, 1994.

CARLZON, JEAN. El Momento de La Verdad. ED. Díaz de Santos, BUENOS AIRES 1991, 147 PP.

ALAIN, SPAEY. La gastronomía y el turismo hoy. pp. $20-45,2004$.

Introducción general a la gestión de la calidad. 2003.

Guía para la aplicación de un programa haccp industria española.

Buenas prácticas de manipulación de alimentos en restaurantes -guía del consultor- prompyme, 2005.

Restaurantes, una guía para mejorar su empresa con base en la productividad y la calidad de trabajo. Organización internacional del trabajo -secretaría de trabajo y previsión social 17 de febrero de 2000 p. 30.

Guía práctica de seguridad sanitaria alimentaria de servicios de alimentación. José Palomino. H. -Aplicación del sistema de análisis de riesgos en puntos críticos de control haccp en el control sanitario de alimentos y en la supervisión de servicios de alimentación en restaurantes, hoteles y servicios similares. 
HOROWITZ, J Y JURGENS PANAK, M. La satisfacción total del cliente. ED. MC GRAW HILL.

GUTIERREZ, M. Nociones de calidad total. ED. LIMUSA, 1997.

FORUM QALITAT, Documentos de gestión 90 casos prácticos. ED. MILENIO, 1997.

Club gestión de la calidad, NUEVO MODELO EUROPEO DE LA EXCELENCIA, 1999.
ASOC. Japonesa de Relaciones Humanas, El libro de las ideas para producir mejor. ED. GESTIÓN 2000.

UGO FEA. Competitividad es calidad total.ED. MARCOMBO, 1993.

ABC. La partición del personal en la mejora continúa de las empresas.

F.J. LLORENS Y M.M. FUENTES. Calidad Total. Fundamentos e Implantación. ED. PIRÁMIDE, 2000. 